\title{
Fördernde Wirkungen von Carnosin ( $\beta$-Alanyl-l-Histidin) auf die Granulation und die Wundheilung
}

\author{
Von \\ Kineshiro NAGAI,* Hiroshi KODAIRA**, Hiroshi KABUTAKE**, \\ Hajime TAKANO** und Tadaharu OKI**
}

\section{Einleitung}

Garnosin ist das $\beta$-Alanyl-1-Histidin, welches das im Jahre 1900 von GuLEwITSCH et al. aus dem Fleischsaft herausgefundene Peptid darstellt. Es ist Zugegen vor allem im Skelettmuskel der Säugetiere. Das $\beta$-Alanyl-1-Methyl-Histidin (Anserin) bzw. das $\beta$-Alanyl-3-Methyl-Histidin (Ophidin) ist andererseits vor allem in Vögeln bzw. in Schlangen ausgesucht. Über die echte physiologische Bedeutung der Existenz des Carnosin gehen die Ansichten noch sehr auseinander, obgleich physiologische, biochemische und pharmakologische Untersuchungen über die Bedeutung von Existenz des Carnosin seit seiner Erfindung bis auf heute aufgeführt sind.

Im Jahre 1967 gingen NAGAI und seine Mitarbeiter ${ }^{1,4), 7)}$ in die Wirkungen von verschiedenen $\omega$-Aminosäuren auf Entzündungen ein und fanden heraus, dass die 4Hydroxy-3-Aminobuttersäure sowie die 4-Amino-3-Hydroxybuttersäure gerade die ausschwemmende Wirkung gegen Carageeninödem und, an Hand der Formalin-FilterPapier-Methode, die entzündlich-granulations-fördernde Wirkung repräsentieren.

Unter der Voraussetzung, dass gewisse chemische Vermittler für die Reparation des entzündeten Gewebes in der Nähe dieser $\omega$-Aminosäuren liegen, fand $\mathrm{NAGAI}^{2,3}$, 5),6) im Jahre 1970 heraus, dass Carnosin von ähnlicher Wirkung wie diese $\omega$-Aminosäuren ist. Seine wirksame Dosis beträgt ca. $25 \mathrm{mg} / 100 \mathrm{~kg}$ Ratte, und zwar ca. ein Hundertstel vom gewebseigenen Carnosin-Gehalt.

Bei der Wundheilung ist die Proliferation des Granulations-granulationsgewebes immer vorausgesetzt. Die Autoren laben eine Nachprüfung der Wirkungen von Carnosin auf die Proliferation des bei der Formalin-Filter-Papier-Methode auszubildenden entzündlichen Granulationsgewebes angestellt, und, anhand von Heilversuch bei der Schnittwunde-Methode, die heilungsfördernde Wirkung von Carnosin sichergestellt, worüber hier ausführlich berichtet werden soll.

\section{Versuchsmethode}

\subsection{Versuchstiere}

In den Granulationsversuch nach der Formalin-Filter-Papier-Methode bzw. den

\footnotetext{
* Physiologisches Institut, Nihon Universität Zahnärztliche Fakultät, Tokyo.

** Physiologische Abteilung, Nihon Universität Zahnärztliche Hochschule zu Matsudo, Matsudo.
} 
Wundheilungsversuch wurden 7 bzw. 5 männliche Wister-Ratten im Durchschnittsgewicht von $100 \mathrm{~g}$ bzw. $120 \mathrm{~g}$ genommen.

\subsection{Versuchsmittel}

Als Versuchsmittel dienten Carnosin in Form von $\beta$-Alanyl-1-Histidin und 1Histidin- $\mathrm{HCl}$ sowie $\beta$-Alanin zur Kontrolle von gleichem Mol wie bei Carnosin.

1) Formalin-Filter-Papier-Methode;

Das Filterpapier, TOYO-Nr. 126 wurde zu Scheiben von $7 \mathrm{~mm}$ Diameter abgeschnitten, mit der 7\% Formalin-Lög. getränkt und in die subkutanen Gewebe im Gebiete der Scapula begrabt. Die Tiere wurden 7 Tage nach dem Begraben geschlachtet; das Filterpapier mitsamt den umgebenden Granulationsgewebe wurde daraus entnommen und im ganzen gewogen. In der Dosierung von Carnosin ging man so vor, dass $0.2 \mathrm{ml}$ von je 70,60, 50, 40, 30, 25, 20, 15 und $10 \mathrm{mg} / \mathrm{kg}$ Carnosin enthaltender Aqu.dest.-Lösung intraperitoneal verabreicht wurden. Die erstmalige Injektion wurde innerhalb 30 Minuten nach dem Begraben gemacht; dann wurde es 6mal alle 24 Stunden nachgespritzt und die Tiere wurden 24 Stunden nach dem allerletzten Spritzen geschlachtet.

\subsection{Wundheilungsversuch}

Man verwundete dem Tier die Bauchwand durch Schneiden auf $20 \mathrm{~mm}$ Länge und $1 \mathrm{~mm}$ Tiefe caudalwärts von unterhalb des Schwertfortsatzes des Brustbeins. Die naturgemässe Aufklappbreite unmittelbar nach sowie 72 Stunden nach dem Schneiden und die Aufklappbreite nach 10 Minuten Auseinanderziehen der Wundränder mit je $10 \mathrm{~g}$ Belastung wurden bestimmt. Die Wunde wurde nach der Bestimmung der naturgemässen Aufklappbreite unmittelbar nach dem Schneiden in ihrer Mitte einmal genäht, und die Bestimmung nach 72 Stunden wurde erst nach der Entfernung der Naht vorgenommen.

Anhand von den Messwerten wurden der Abschlussindex I bei naturgemässer Schnittwundheilung und der Abschlussindex II unter Belastung durch Auseinanderziehen der Wundränder aus den Folgenden Formeln Errechnet:

$$
\begin{aligned}
\text { Abschlussindex I }= & \frac{\text { Naturgemässe Aufklappbreite - }}{\text { Naturgemässe }} \\
& \frac{\text { Naturgemässe Auf klappbreite nach } 72 \mathrm{Stdn} . \%}{\text { Auf klappbreite }} \% \\
\text { Abschlussindex II }= & \frac{\text { Belastungsauf klappbreite- }}{\text { Belastungsauf klappbreite }} \\
& \text { Belastungsaufklappbreite nach } 72 \mathrm{Stdn} . \%
\end{aligned}
$$

\section{Ergebnisse}

1) Granulationsfördernde Wirkung von Carnosin, 1-Histidin, $\beta$-Alanin und 1-Histidin $+\beta$-Alanin, geprüft nach der Formalin-Filter-Papier-Methode; Die Untersuchungsresultate finden sich in folgenden Tabellen. 
Tabelle 1 Wirkung von Carnosin auf die Granulationsbildung

\begin{tabular}{lcc}
\hline Dosis & $\begin{array}{c}\text { Anzahl der } \\
\text { Granulation }\end{array}$ & $\begin{array}{c}\text { Gewicht von } \\
\text { Granulation (Mittelwerte) } \pm \text { SD }\end{array}$ \\
\hline $70 \mathrm{mg} / \mathrm{kg}$ ip & 14 & $178.3 \mathrm{mg} \pm 4.8^{*}$ \\
60 & 14 & $173.3 \pm 5.2^{*}$ \\
50 & 14 & $174.0 \pm 5.8^{*}$ \\
40 & 14 & $178.0 \pm 7.9^{*}$ \\
30 & 14 & $170.5 \pm 10.1^{*}$ \\
25 & 14 & $169.7 \pm 2.3^{*}$ \\
20 & 14 & $159.4 \pm 4.1^{*}$ \\
15 & 14 & $150.8 \pm 6.1$ \\
10 & 14 & $148.0 \pm 6.2$ \\
\hline Kontrolle & 20 & $140.5 \pm 9.2$ \\
\hline
\end{tabular}

Die Granulationsbildung ging, wie es aus den Versuchsergebnissen ersichtlich ist, fast in entsprechenden Dosen vor sich, und zwar in der wirksamen Mindestdosis $20 \mathrm{mg} / \mathrm{kg}$ ip. und darüber. In Dosen von mehr als $40 \mathrm{mg} / \mathrm{kg}$ war es nicht bei weitem wirksam als von 50,60 und $70 \mathrm{mg} / \mathrm{kg}$.

Tabelle 2 Wirkung von 1-Histidin auf die Granulationsbildung

\begin{tabular}{lcc}
\hline \hline Dosis & $\begin{array}{c}\text { Anzahl der } \\
\text { Granulation }\end{array}$ & $\begin{array}{c}\text { Gewicht von } \\
\text { Granulation (Mittelwerte) }\end{array}$ \\
\hline $27 \mathrm{mg} / \mathrm{kg}$ & 14 & 138.0 \\
20 & 14 & 140.8 \\
17 & 14 & 140.1 \\
13 & 14 & 136.4 \\
10 & 14 & 141.0 \\
\hline Kontrolle & 14 & 139.2 \\
\hline
\end{tabular}

Die Granulationsbildung in den Fällen von 1-Histidin Verabreichung, wie es aus der Tabelle ersichtlich ist, liess keinerlei signifikante Abweichungen erkennen von derselben in den Kontrollfällen.

Tabelle 3 Wirkung von $\beta$-Alanin auf die Granulationsbildung

\begin{tabular}{lcc}
\hline \hline Dosis & $\begin{array}{c}\text { Anzahl der } \\
\text { Granulation }\end{array}$ & $\begin{array}{l}\text { Gewicht von } \\
\text { Granulation (Mittelwerte) }\end{array}$ \\
\hline $15 \mathrm{mg} / \mathrm{kg}$ & 14 & 130.1 \\
11 & 14 & 138.4 \\
\hline Kontrolle & 14 & 132.5 \\
\hline
\end{tabular}

Die Granulationsbildung in den Fällen von $\beta$-Alanin-Verabreichung, wie es die Tabelle wiedergibt, liess keinerlei signifikante Abseichungen erkennen von derselben in den Kontrollfällen. 
Tabelle 4 Beeinflussung der Granulationsbildung aurch die kombinierte Verabreichung von 1-Histidin und $\beta$-Alanin

\begin{tabular}{llcc}
\hline 1-Histidin & -Alanin & $\begin{array}{c}\text { Anzahl der } \\
\text { Granulation }\end{array}$ & $\begin{array}{c}\text { Gewicht von } \\
\text { Granulation (Mittelwerte) }\end{array}$ \\
\hline $27 \mathrm{mg} / \mathrm{kg}$ & $15 \mathrm{mg} / \mathrm{kg}$ & 14 & 150,4 \\
20 & 11 & 14 & 146,8 \\
\hline Kontrolle & & 14 & 148,0 \\
\hline
\end{tabular}

Die Granulationsbildung in den Fällen von kombindierter Verabreichung von $\beta$-Alanin und 1-Histidin, wie es sich aus den obigen Daten ergibt, liess keinerlei signifikante Abweichungen erkennen von derselben in den Kontrollfällen.

Tabelle 5 Auswertung von Carnosin-Wirkung durch Abschlussindex nach 72 Stunden

\begin{tabular}{|c|c|c|c|c|}
\hline & \multicolumn{2}{|c|}{ Abschlussindex I } & \multicolumn{2}{|c|}{ Abschlussindex II } \\
\hline & $\begin{array}{l}\text { naturgemässe } \\
\text { Aufklappbreite } \\
\text { (mm) beim } \\
\text { Schneiden } \\
(\mathrm{M} . \mathrm{W} . \pm \mathrm{SE} \text { ) }\end{array}$ & $\begin{array}{l}\text { naturgemässe } \\
\text { Aufklappbreite } \\
\text { (mm) bei Ent- } \\
\text { fernung der } \\
\text { Naht nach } 72 \\
\text { Stunden } \\
\text { (M.W. } \pm \text { SE) }\end{array}$ & $\begin{array}{l}\text { Belastungs- } \\
\text { aufklappbreite } \\
(\mathrm{mm}) \text { beim } \\
\text { Schneiden } \\
(\mathrm{M} . \mathrm{W} . \pm \mathrm{SE})\end{array}$ & $\begin{array}{l}\text { Belastungs- } \\
\text { aufklappbreite } \\
\text { (mm) nach } 72 \\
\text { Stuoden } \\
(\mathrm{M} . \mathrm{W} . \pm \mathrm{SE} \text { ) }\end{array}$ \\
\hline Kontrolle & \multicolumn{2}{|c|}{37,1} & \multicolumn{2}{|c|}{36,3} \\
\hline $40 \mathrm{mg} / \mathrm{kg}$ Carnosin & $3,5 \pm 0,65$ & $2,2 \pm 0,21$ & $16,5 \pm 0,6$ & $10,5 \pm 0,30$ \\
\hline $25 \mathrm{mg} / \mathrm{kg}$ Carnosin & \multicolumn{2}{|c|}{85,1} & \multicolumn{2}{|r|}{ 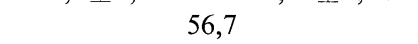 } \\
\hline $27 \mathrm{mg} / \mathrm{kg} \mathrm{1-Histidin}$ & \multicolumn{2}{|c|}{41,1} & \multicolumn{2}{|c|}{42,0} \\
\hline $\begin{array}{c}27 \mathrm{mg} / \mathrm{kg} \text { Histidin } \\
+\end{array}$ & \multicolumn{2}{|c|}{39,0} & \multicolumn{2}{|c|}{42,6} \\
\hline $15 \mathrm{mg} / \mathrm{kg} \beta$-Alanin & $4,1 \pm 0,41$ & $2,5 \pm 0,61$ & 17,8 & $9,8 \pm 0,31$ \\
\hline
\end{tabular}

\section{Betrachtung}

Bei der Förderung der entzündlichen Granulation betrug die wirksame Dosis $20 \mathrm{mg} / \mathrm{kg}$ ip nach der Formalin-Filter-Papier-Methode. Die Granulationsvolumen nahm mit der Dosensteigerung zu, erreichte ihr Maximum in der Dose von $40 \mathrm{mg} / \mathrm{kg}$, und zeigte nur sehr wenig Neigung zur weiteren Volumenzunahme in den Dosen von $60 \sim 50 \mathrm{mg} / \mathrm{kg}$, nicht viel anders als in der Dose von $40 \mathrm{mg} / \mathrm{kg}$. Diese Tatsache beweist, dass Carnosin in seinem Überschuss ohen Einfluss bleibt, da ebensoviel Carnosin die Reparatur abzuschliessen vermag, als das Moment für die Entzündung liegen soll, welchem das Mass der gebildeten Granulation proportional ist. In der Annahme, dass die Wirkung von Carnosin auf die Granulation im Grunde auf die Aufbauaminosäuren herruhen könnte, wurden die Wirkungen von $\beta$-Alanin-1-Histidin von gleichem mol wie bei dem verabreichten Carnosin und von dem Gemenge dieser beiden Sub- 
stanzen auf die Granulation nach der Formalin-Filter-Papier-Methode bestimmt. Die Werte der Wirkungen von den Aufbauaminosäuren und dem Gemenge, wie as sich aus den Tabellen 2, 3 und 4 ergibt, unterschieden sich nicht signifikant von den Kontrollwerten.

Die wirksame Dosis betrug $20 \mathrm{mg} / \mathrm{kg}$, und zwar $2 \mathrm{mg}(8.8 \mu \mathrm{mol}) / 100 \mathrm{~g}$ Ratte, und es entspricht einem 1/35 des Carnosin-Gehaltes des M. gastrocnemius der Ratte (308 $\mu \mathrm{mol} / 100 \mathrm{~g})$. Als die chemischen Vermittler der entzündlichen Exsudation sind mancherlei Substanzen wie Ach, Serotonin, Histamin, Bradykinin, SRS, usw. identifiziert, jedoch sind diejenigen Vermittler der Reparatur der Entzündung noch unbekannt, welche Vorgänge angeblich von der entzündlichen Exsudation qualitativ und quantitativ beherrscht werden. Im Jahre 1970 fanden NAGAI und seine Mitarberiter nachweislich heraus, dass die reparationsfördernde Wirkung auf das Gewebe dem Carnosin, besonders in Verbindung mit Cortison, zukommt. Daher führten sie zu der Annahme, dass Cortison physiologisch nicht entzündungshemmend zur Wirkung zu kommen, sondern sich nur bei Anwesenheit der Co-Faktoren wie z.B. Carnosin an der Abwehrreaktion zu beteiligen vermag, und bezeichneten diese Co-Faktoren als PRIS (Protective Response Inducing Substance $=$ Abwehrreaktions-erzeugende Substanzen).

Da Carnosin schon in Dosen von. $8.8 \mu \mathrm{mol} / 100 \mathrm{~g}$ Ratte, der pharmakologischwirksamen Dosis für die Granulationsreparatur, wirksam ist, und dementsprechend die tatsächliche Wirkungsdosis auf dem Entzündungssitz viel weniger als $8.8 \mu \mathrm{mol}$ ist, soll die Wirkungsdosis von Carnosin als dem von NaGAI genannten Vermittler der Reparatur (PRIS) minimal sein. Die Fähigkeit von Carnosin, in weitaus gerigeren Dosen als im physiologischen Soll-Gehalt zu wirken, hat als die reparatorische Funktion des chemischen Vermittlers eine grosse Bedeutung gelangt.

\section{Heilwirkung auf Schnittwunde}

Carnosin war in Dosen von $40 \mathrm{mg} / \mathrm{kg}$ ip sowie $25 \mathrm{mg} / \mathrm{kg}$ ip, wie die Versuchsergebnisse in der Tabelle 5 zeigen, imstande, den Abschlussindex I und II zu verbessern, verglichen mit demjenigen nach der Verabreichung von den Aufbauaminosäuren von gleichem Mol wie bei den verwendeten Carnosin sowie von dem Gemenge dieser beiden Substanzen.

Der Abschlussindex I zeigt die gewöhnliche Heilwirkung, während der Abschlussindex II den Stärkegrad der Verklebung der Schnittwunde repräsentiert, so dass die Dichtigkeit der Abschlusszustande der Wunde ermittelt werden kann. Dies ist eine gleichartige Methode wie Dispasqualesche Methode der Auswertung von Pharmaka durch Messung von "tens ile strengter" in der Schnittstelle.

Die Versuchsergebnisse in der Tabelle 5 sind imstande, zu der granulationsfördernden Wirksamkeit von Carnosin nach der Formalin-Filter-Papier-Methode zu ergänzen.

\section{Schlussfolgerung}

1. Carnosin ist bei der Granulationsreparatur nach der Formalin-Filter-PapierMethode von Wirksamkeit, u.z.w. in Dosen von $20 \mathrm{mg}(8.8 \mu \mathrm{Mol}) / 100$ Ratte. 
2. Die Granulationsförderung durch Carnosin erreichte in Dosen von $40 \mathrm{mg} / \mathrm{kg}$ ip ihr Maximum und ging nicht weiter sogar in Dosen üter $40 \mathrm{mg} / \mathrm{kg}$ ip hinaus.

3. Carnosin erwies sich bei der Heilung der Schnittwunde als wirksam mit höheren Abschlussindex I und II als bei den Kontrollfällen.

(Eingang 5. Dez. 1973)

\section{Literatur}

[1] NaGAI, K. et al.: Synergistic Wirkung des Cortison-GABOB-Systems im Abwehmechanisms, Jap. J. of Brain physiolog., 106: 75-80, 1969.

[2] NAGAI, K. et al.: Die physiologishe Bedeutung von Carnosin bei der Inflammation, ArzneimForsch. (Drug Res.) 20: 12, 1876-1978, 1970.

[3] NaGAI, K. et al.: Die hemmende Wirkung von Carnosin auf verschiedene allergische Reaktionen, Arzneim.-Forsch. (Drug Res.) 21: 1222-1225, 1971.

[4] NagaI, $K$. et al.: Studies on the synergistic action and antiulcerous activity of cortisonGABOB, Arzneim.-Forsch. (Drug Res.) 21: 96-97, 1971.

[5] NAGAI K.: Physiological implications of carnosine on the inflammation-with reference to its inhibitory action of allergy and the vital defense mechanism- J. Nihon Univ. Sch. Dent: 13: 1, 1-12, 1971.

[6] NaGAI K.: Erklärung auf dem physiologischen Mechanismus von Inframmation, besonders bei Reparation, J. Nihon Univ. Sch. Dent., 14: 2, 42-52, 1972.

[7] NAGAI, K. et al.: Kliniche Wirksamkeit von 4-Hydroxy-3-Amino Buttersäure (GOBAB) bei Dekubitsu, J. Nihon Univ. Sch. Dent., 14: 2, 53-57, 1972. 